\title{
Understanding the role of key point Mutations in Receptor Binding Domain of SARS-CoV-2 Spike Glycoprotein \\ Bipin Singh*
}

Department of Applied Sciences, BML Munjal University, Gurugram-122413, Haryana, India

*E-mail-bipin.singh@bmu.edu.in

\begin{abstract}
The recent outbreak of novel coronavirus (2019-nCoV or SARS-CoV-2) and its spread to the whole world is currently posing one of the major threat to human health and the world economy. It has been suggested that 2019-nCoV is similar to SARS-CoV based on the genome sequence comparison. Despite the genomic similarity between SARS-CoV and SARS-CoV-2, the spike glycoprotein and receptor binding domain in SARS-CoV-2 shows considerable difference compared to SARS-CoV, due to the presence of several point mutations. We analyzed the receptor binding domain (RBD) from recently published 3D structure of spike glycoprotein of SARS-CoV-2 and compared with RBD of SARS-CoV. The observations highlight few important features of RBD in the light of the recently published findings from the 3D structures of spike glycoprotein and its complex with human angiotensin-converting enzyme 2 (ACE2) (Yan, R., et al. (2020); Wrapp, D., et al. (2020); Walls, A. C., et al. (2020)).
\end{abstract}

Keywords: Covid-19, SARS-CoV-2, ACE2, Coronavirus, 2019-nCoV

\section{INTRODUCTION}

The recent outbreak of Covid-19 caused by SARS-CoV-2 virus has been declared as pandemic by World Health Organization and currently spread to around 190 countries across the world. Similar to SARS-CoV, the SARS-CoV-2 uses angiotensin-converting enzyme 2 (ACE2) on surface membrane of humans cells for invasion [2-4]. The interaction between viral spike glycoprotein and ACE2 on the host cell surface is important from the point of view of development of therapeutics and drugs, as it initiates the process of infection [5]. The recently published research papers provided the 3D molecular structure of SARS-CoV-2 spike glycoprotein and complex of spike glycoprotein and human ACE2 [2-4]. Here we present a comparative analysis of receptor binding domain of SARS-CoV and SARS-Cov-2 using the published 3D structures and highlight the role of few key point mutations in receptor binding domain of spike glycoprotein that seems important for observed efficient binding to ACE2.

\section{RESULTS AND DISCUSSIONS}

\section{Molecular level explanation for enhanced binding affinity of RBD of SARS-CoV-2 to Human ACE2}

It was shown from the previous studies that the mutations in receptor binding domains (RBD) of spike glycoprotein of SARS-CoV have important implications in understanding the cross species transmission of virus [1]. Therefore, comparison of RBD of SARS-CoV and SARS-CoV-2 has been performed to get insights of how the RBD has evolved in SARS-CoV-2 compared to SARS-CoV.

The previous research has suggested that residues Leu472, Asn479 and Thr487 critical for cross-species and human to human transmission of SARS-CoV [1]. In SARS-CoV-2 these residues are mutated to Phe $486, \mathrm{Gln} 493$ and Asn501 respectively. It is interesting to note that all these three point mutations seems to have dual benefit for SARS-CoV-2, i.e., preserving 
the physicochemical nature of side chain and increasing the surface area for efficient interaction with human ACE2. Therefore, we can speculate that these mutations can provide the SARS-CoV-2 a better ability of human-to-human transmission compared to SARS-CoV.

The mutations in RBD of SARS-CoV-2 such as TYR442 $\rightarrow$ LEU455, LEU443 $\rightarrow$ PHE456, PHE460 $\rightarrow$ TYR473, PRO462 $\rightarrow$ ALA475, LEU472 $\rightarrow$ PHE486 and ASN479 $\rightarrow$ GLN493 [2] tends to increase the hydrophobic side chain surface area through the formation of non-covalent interaction network (Figure 1). Furthermore, the mutation THR487 $\rightarrow$ ASN501 tends to increase the polar side chain surface area. We hypothesize that the mutation ASP463 $\rightarrow$ GLY476 and TYR484 $\rightarrow$ GLN498 in RBD of SARS-CoV-2 may provide the required flexibility in loop region that could be important in formation of this extended non-covalent interaction network.

The analysis of interface between RBD of SARS-CoV-2 and human ACE2 shows that the multiple mutations in RBD have helped in reducing the volume of cavity in SARS-CoV-2 compared to SARS-CoV (Figure 2). The reduction in cavity volume might help in enhanced binding of SARS-CoV-2 to ACE2 that was observed experimentally [4].

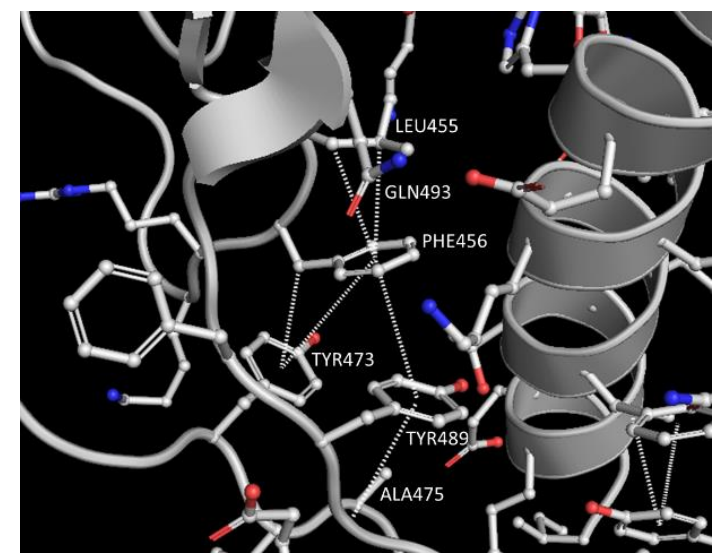

Sars-CoV-2

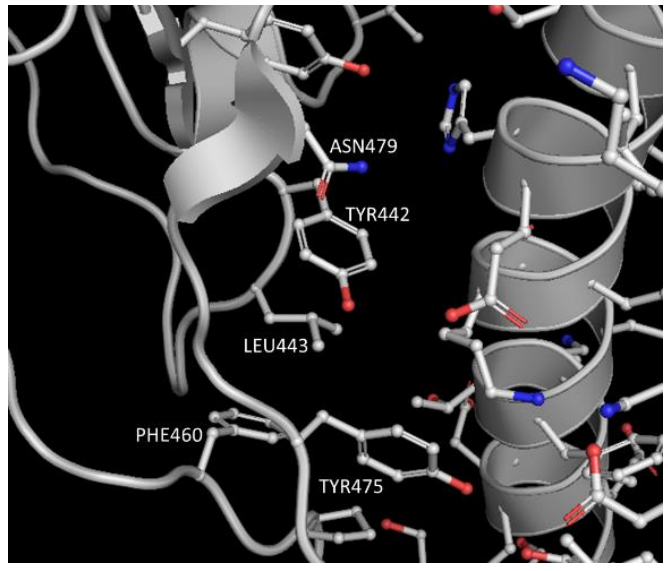

Sars-CoV

Figure 1. Comparative analysis of impact of point mutations in SARS-CoV-2 with reference to SARS-CoV. 


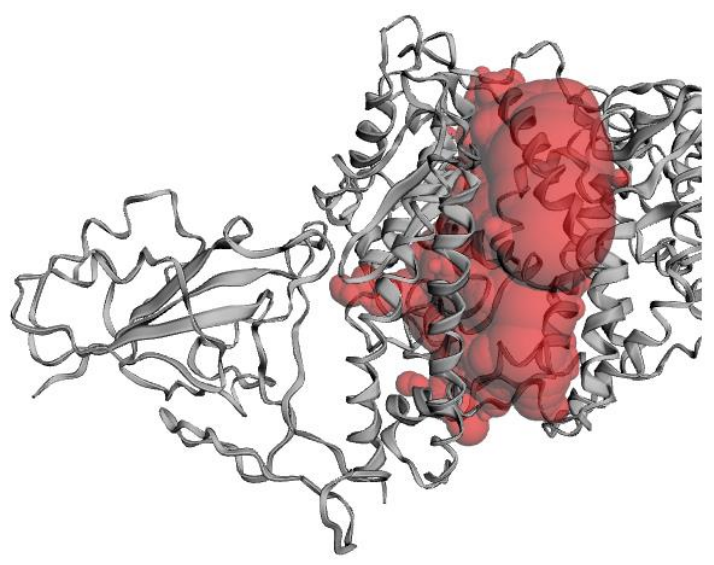

SARS-CoV-2

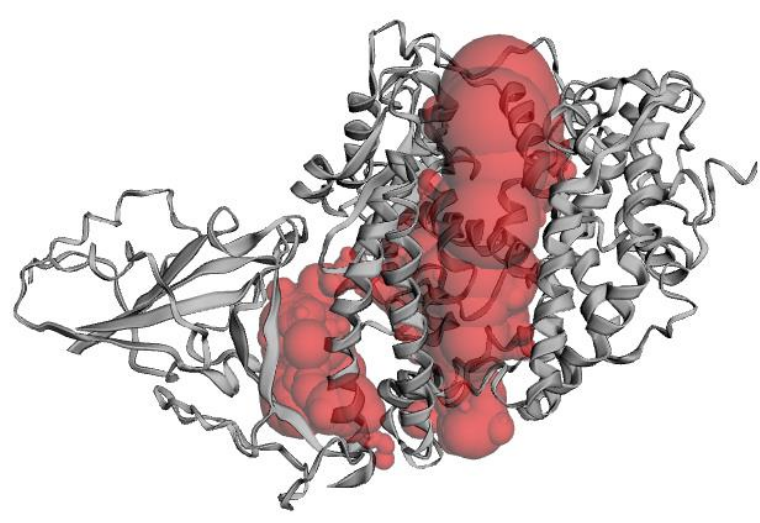

SARS-CoV

Figure 2. Comparison of pockets at the binding interface of RBD and ACE2 in the 3D structures of SARS-CoV-2 (PDB ID: 6M17) and SARS-CoV (PDB ID: 2AJF).

\section{CONCLUSIONS}

The network of non-covalent interactions formed due to key point mutations in RBD region of spike glycoprotein of SARS-CoV-2 could be an interesting target for drug discovery, since disrupting the network of non-covalent interactions at the interface of RBD and ACE2 could lead to weakening of binding of SARS-CoV-2 to ACE2 in humans and therefore can be a useful strategy for the development of novel therapeutics and drugs against Covid-19.

\section{METHODOLOGY}

The coordinates of the 3D structures of SARS-CoV [1] and SARS-CoV-2 [2] were taken from protein data bank [6]. For calculating of non-covalent interactions, we used the Arpeggio web server [7]. The pocket analysis was performed using the Castp 3.0 web server [8]. The figures were prepared using Pymol molecular visualization program [9].

\section{REFERENCES}

1. Li, F., Li, W., Farzan, M., \& Harrison, S. C. (2005). Structure of SARS coronavirus spike receptor-binding domain complexed with receptor. Science, 309(5742), 1864-1868.

2. Yan, R., Zhang, Y., Li, Y., Xia, L., Guo, Y., \& Zhou, Q. (2020). Structural basis for the recognition of the SARS-CoV-2 by full-length human ACE2. Science.

3. Wrapp, D., Wang, N., Corbett, K. S., Goldsmith, J. A., Hsieh, C. L., Abiona, O., ... \& McLellan, J. S. (2020). Cryo-EM structure of the 2019-nCoV spike in the prefusion conformation. Science, 367(6483), 1260-1263.

4. Walls, A. C., Park, Y. J., Tortorici, M. A., Wall, A., McGuire, A. T., \& Veesler, D. (2020). Structure, function, and antigenicity of the SARS-CoV-2 spike glycoprotein. Cell. 
5. Liu, C., Zhou, Q., Li, Y., Garner, L. V., Watkins, S. P., Carter, L. J., ... \& Albaiu, D. (2020). Research and Development on Therapeutic Agents and Vaccines for COVID-19 and Related Human Coronavirus Diseases. ACS Central Science. DOI: 10.1021/acscentsci.0c00272. DOI: 10.1021/acscentsci.0c00272

6. Stephen K Burley, Helen M. Berman, et al (2019). RCSB Protein Data Bank: biological macromolecular structures enabling research and education in fundamental biology, biomedicine, biotechnology and energy Nucleic Acids Research 47: D464-D474.

7. Jubb, H. C., Higueruelo, A. P., Ochoa-Montaño, B., Pitt, W. R., Ascher, D. B., \& Blundell, T. L. (2017). Arpeggio: a web server for calculating and visualising interatomic interactions in protein structures. Journal of molecular biology, 429(3), 365-371.

8. Tian, W., Chen, C., Lei, X., Zhao, J., \& Liang, J. (2018). CASTp 3.0: computed atlas of surface topography of proteins. Nucleic acids research, 46(W1), W363-W367.

9. DeLano, W. L. (2002). The PyMOL molecular graphics system. http://www. pymol. org. 